\title{
Fatores de risco para sobrepeso entre adolescentes: análise de três cidades brasileiras
}

\author{
Risk factors for overweight among adolescents: \\ analysis of three Brazilian cities
}

Isabela da Costa RIBEIRO'

Fernando Antonio Basile COLUGNATI ${ }^{2}$

José Augusto de Aguiar Carrazedo TADDEI

\section{RE S U M O}

\section{Objetivo}

Realizar análise comparativa de fatores de risco para sobrepeso em adolescentes de três cidades brasileiras, Pelotas, São Paulo e Goiânia.

\section{Métodos}

Os três bancos de dados são provenientes de estudos com delineamento do tipo caso-controle, que investigaram fatores de risco para sobrepeso/obesidade entre adolescentes das três cidades. Classificaram-se os adolescentes como sobrepeso quando o Índice de Massa Corporal estava acima do percentil 85 em relação ao padrão de referência do National Center for Health Statistcs. Procedeu-se à análise multivariada com modelo hierárquico de regressão logística.

\section{Resultados}

Foram estudados 903 adolescentes pós-púberes (443 casos e 460 controles). A análise não-ajustada dos três bancos de dados combinados aumentou o poder das associações quando meninos e meninas foram considerados separadamente. Educação materna menor ou igual a 8 anos duplicou o risco de sobrepeso entre meninas; obesidade dos pais esteve fortemente associada ao sobrepeso dos adolescentes; tempo de televisão maior ou igual a 4 horas associou-se ao sobrepeso apenas entre meninas; consumo de gordura visível da carne aumentou o risco de sobrepeso entre os meninos em $70 \%$ e consumo de frituras apresentou associação inversa entre as meninas. Finalmente, o consumo de álcool esteve significante e inversamente associado ao sobrepeso entre meninos.

\section{Conclusão}

Meninas pós-púberes, provenientes de famílias com baixa escolaridade, devem receber atenção especial durante o planejamento e a execução de estratégias de prevenção e controle do sobrepeso.

Termos de indexação: Adolescente. Epidemiologia analítica. Índice de massa corporal. Sobrepeso.

\footnotetext{
1 Pontifícia Universidade Católica do Paraná, Centro de Ciências Biológicas e da Saúde, Curso de Nutrição. Curitiba, PR, Brasil.

2 Instituto de Pesquisas em Tecnologia e Inovação. São Paulo, SP, Brasil.

3 Universidade Federal de São Paulo, Departamento de Pediatria. R. Loefgreen, 1647, Vl. Clementino, 04040-032, São Paulo, SP, Brasil. Correspondência para/Correspondence to: J.A.A.C. TADDEI.E-mails: <taddei.dped@epm.br>; <nutsec@yahoo.com.br>.
} 
504 | I.C. RIBEIRO et al.

\section{A B S T R A C T}

\section{Objective}

The objective of this study was to conduct a comparative analysis of risk factors for overweight among adolescents from three Brazilian cities, Pelotas, São Paulo and Goiânia.

\section{Methods}

The three datasets derived from case-control studies that investigated risk factors for overweight/obesity among adolescents from the three cities. Adolescents were classified as overweight when body mass index exceeded the $85^{\text {th }}$ percentile of the National Center for Health Statistics. Multivariate analysis using a hierarchical logistic regression model was performed.

\section{Results}

A total of 903 postpubertal adolescents were studied (443 cases and 460 controls). The unadjusted analysis combining the 3 datasets improved the power of associations when males and females were addressed separately. Maternal education $\leq 8$ years doubled the risk of overweight among girls; parents' obesity was a strong predictor of adolescents' overweight; watching television for $\geq 4$ hours was significantly associated with females' overweight but not males'; consumption of visible fat from meat increased the risk of overweight among boys by $70 \%$ while the consumption of fried foods had a negative association with girls' body mass index. Finally, alcohol consumption was significantly and inversely associated with overweight among boys only.

\section{Conclusion}

Postpubertal girls from families with lower educational levels should receive special attention when strategies to prevent and control overweight are planned and executed.

Indexing terms: Adolescent. Analytic Epidemiology. Body mass index. Overweight.

\section{N T R O D U ÇÃ O}

A adolescência é um período de mudanças intensas na vida de qualquer indivíduo. O rápido crescimento linear, associado a alterações hormonais, cognitivas e emocionais, faz com que a adolescência seja considerada um período da vida especialmente vulnerável do ponto de vista da nutrição' ${ }^{1}$. E também fase de eleição para a incorporação de medidas preventivas, uma vez que, em função de modismos, propaganda, escola, amigos e contestação de valores familiares e sociais, os adolescentes podem modificar seus hábitos alimentares².

A prevalência mundial de obesidade vem aumentando rapidamente, sendo considerada uma epidemia nos países desenvolvidos. Em países em desenvolvimento, experimentando acelerado processo de urbanização, a obesidade é, ao lado de outros agravos ligados ao subdesenvolvimento, um dos maiores problemas de saúde pública ${ }^{3,4}$. No Brasil, a análise das informações obtidas em quatro inquéritos nutricionais (1974/1975, 1989,
1996/1997 e 2002/2003) demonstra intenso aumento na prevalência de sobrepeso entre adolescentes, afetando-os de forma diferenciada, segundo região estudada, sexo e faixa etária 5 . 0 último desses inquéritos estima entre 18,0 e $15,4 \%$ o excesso de peso no país entre adolescentes de 10 a 19 anos $^{6}$.

O predomínio de fatores de risco relacionados à dieta, à atividade física e ao sobrepeso/obesidade aumenta, à medida que o estilo de vida dos brasileiros fica mais parecido com o dos países desenvolvidos. Esses fatores de risco elevam os riscos de doenças crônicas como diabetes, hipertensão, doenças cardiovasculares, infarto agudo do miocárdio e certas formas de câncer? .

As implicações econômicas dessas mudanças no perfil epidemiológico da população mundial já são visíveis e profundas, seja do ponto de vista do comprometimento da saúde daqueles que representam mão-de-obra necessária para o contínuo desenvolvimento dos países como dos gastos diretos e indiretos com serviços médicos e 
atenção à saúde em geral ${ }^{8}$. Sichieri \& Nascimento ${ }^{9}$ demonstram, em seu estudo, que as despesas com hospitalização por obesidade/sobrepeso como causa direta não representam grande impacto nos custos hospitalares no Brasil. No entanto, em 2001 a proporção de despesas com as co-morbidades de obesidade e sobrepeso foram equivalentes a $3,02 \%$ e $5,83 \%$ dos custos totais de hospitalização para homens e mulheres, respectivamente ${ }^{9}$.

Os dados apresentados a seguir são provenientes de três cidades brasileiras experimentando diferentes estágios de transição epidemiológica e nutricional em função das diversas fases dos processos demográficos e de desenvolvimento em que se encontram. O Quadro 1 descreve algumas características demográficas ${ }^{10}$ dessas três cidades, a fim de ilustrar diferenças no que tange à densidade populacional, à longevidade, à escolaridade e à renda. A proposta de análise dos três bancos de dados de forma combinada, utilizando-se técnicas estatísticas adequadas, constitui-se em estratégia para potencializar os efeitos das variáveis independentes sobre o desfecho (sobrepeso entre adolescentes).

Este estudo teve dois propósitos: 1) desenvolver e testar metodologia para a comparabilidade de estudos com questionários diferentes, a partir da manipulação de variáveis compatíveis; 2) proceder à análise comparativa de fatores de risco para sobrepeso entre os três grupos de adolescentes, desenvolvendo modelo de risco para sobrepeso entre os adolescentes estudados segundo o sexo.

\section{MÉ TO D OS}

Apresentam-se a análise de três diferentes bancos de dados provenientes de estudos com delineamento do tipo caso-controle, que investigaram fatores de risco para sobrepeso/obesidade entre adolescentes de Pelotas ${ }^{11}$, São Paulo ${ }^{12}$, e Goiânia ${ }^{13}$.

A fim de garantir a qualidade e a comparabilidade dos estudos a serem combinados foram formulados a priori os seguintes critérios de inclusão $^{14}$ : 1) delineamento tipo caso-controle; 2) coleta de dados antropométricos e entrevistas realizadas por equipes treinadas segundo os mesmos critérios; 3) questionários padronizados e pré-codificados; 4) parecer favorável do Comitê de Ética em Pesquisa da Universidade Federal de São Paulo (Unifesp), n ${ }^{\circ}$ CEP 0472/06. Além destes critérios, ressalta-se que os três estudos foram desenvolvidos junto à Disciplina de Nutrologia, Departamento de Pediatria da Unifesp, garantindo o uso de abordagens de coleta dos dados bastante similares, já que as pesquisadoras receberam treinamento na mesma unidade. Para a medida de peso os entrevistados, vestindo roupas leves e descalços, foram pesados em balança eletrônica com precisão de $100 \mathrm{~g}$. A medida de altura foi realizada com fita métrica inextensível e esquadro de madeira, os adolescentes permanecendo em pé, descalços sobre superfície plana, com os pés unidos e com calcanhares, ombros e cabeça em ângulo reto com essa superfície e olhando para frente.

Quadro 1. Características demográficas das cidades de Pelotas, Goiânia e São Paulo. São Paulo, $2001 / 2002$.

\begin{tabular}{|c|c|c|c|}
\hline Características & Pelotas (RS) & Goiânia (GO) & São Paulo (SP) \\
\hline População em 2001/2002 & 320850 & 1093007 & 10435546 \\
\hline Habitantes $/ 100 \mathrm{~km}^{2}$ & 21,0 & 160,0 & 717,0 \\
\hline Residentes $\geq 65$ anos de idade (\%) & 8,1 & 4,5 & 6,4 \\
\hline Residentes $\geq 10$ anos de idade com menos de um ano de estudo (\%) & 5,0 & 4,0 & 3,9 \\
\hline Residentes $\geq 10$ anos de idade com $\geq 11$ anos de estudo (\%) & 22,7 & 31,9 & 32,1 \\
\hline Residentes $\geq 10$ anos de idade com rendimento mensal até 1 salário-mínimo (\%) & 17,2 & 13,2 & 5,9 \\
\hline Residentes $\geq 10$ anos de idade com rendimento mensal $\geq 10$ salários-mínimos (\%) & 5,2 & 8,3 & 10,8 \\
\hline
\end{tabular}


506 | I.C. RIBEIRO et al.

\section{População em estudo}

\section{Pelotas}

Estudo conduzido na cidade de Pelotas (RS), entre os meses de agosto e dezembro de 2000. Foram pesados e medidos 1608 adolescentes nascidos entre 1/1/1980 e 31/12/1985, os quais representaram $96,6 \%$ dos participantes das aulas de educação física de todas as escolas particulares da cidade de Pelotas nessa faixa etária. Ao final dessa primeira fase foram identificados 264 (16,4\%) adolescentes com sobrepeso (casos), com Índice de Massa Corporal IMC (peso/altura²) igual ou acima do percentil 85, segundo classificação proposta por Must et al. ${ }^{15}$. Para cada indivíduo foi identificado aleatoriamente um controle, pareado por sexo e idade, selecionado entre os indivíduos apresentando IMC entre os percentis 5 e 85, ponto de corte utilizado para garantir que apenas adolescentes eutróficos fossem classificados como controles ${ }^{11}$. Além da entrevista com os adolescentes, houve um contato telefônico com os pais ou responsáveis pelos mesmos, durante o qual foram coletadas informações sobre o adolescente (peso ao nascer, amamentação) e os pais biológicos (peso, altura, escolaridade).

\section{São Paulo}

O estudo de São Paulo ${ }^{12}$ foi desenvolvido como parte do Projeto ECCHOS (Estudos Clínicos sobre Crescimento, Hipertensão Arterial, Obesidade e Saúde Bucal) da Unifesp. Foram pesados e medidos 1420 adolescentes, os quais representam 98,6\% dos estudantes da faixa etária estudada, matriculados em escola pública. A coleta de dados antropométricos e as entrevistas com casos e controles foram realizadas por nutricionistas e pediatras durante o ano de 2002. Além dos adolescentes, pais ou responsáveis também compareceram às entrevistas, nas quais foi possível obter informações sobre o adolescente e sobre a escolaridade e as medidas de peso e estatura dos pais biológicos. Com o intuito de estudar adolescentes apresentando maiores riscos de obesidade, particularmente nesse estudo foram considerados casos os adolescentes com IMC igual ou acima do percentil 89 (15,2\% do total), sendo os controles definidos segundo os mesmos critérios do estudo de Pelotas, pareados por idade, sexo e estágio puberal.

\section{Goiânia}

Estudo conduzido na Universidade Federal de Goiás (UFG), Campus Goiânia entre setembro e dezembro de $2004^{13}$, no qual foram pesados e medidos 1465 estudantes com até 19 anos completos na data de alocação para o estudo, dos quais $8,26 \%$ apresentavam sobrepeso segundo o mesmo critério descrito no estudo de Pelotas. No estudo original de Menezes ${ }^{13}$ foram identificados dois controles para cada caso. As informações obtidas por entrevista foram complementadas por aquelas obtidas em contato telefônico com os pais ou responsável. A fim de atender os propósitos do presente estudo definiu-se a amostra com um controle para cada caso a partir de sorteio dos controles, que foram em seguida pareados por sexo e idade com os casos.

Nos três estudos calculou-se o IMC dos pais a partir do peso e da estatura auto-referidos, considerando-se IMC acima de $30 \mathrm{~kg} / \mathrm{m}^{2}$ indicativo de obesidade ${ }^{16}$. Estudos publicados na literatura internacional são controversos quanto à validade e à confiabilidade do peso e estatura auto-referidos, geralmente relatando superestimação do peso e subestimação da altura, em comparação com valores medidos diretamente ${ }^{17}$. Entretanto, evidências de estudos nacionais ${ }^{18}$ indicam alta sensibilidade e especificidade das informações de peso e altura auto-referidas. Fonseca et al. ${ }^{18}$, estudando 3713 funcionários técnico-administrativos de uma universidade do Rio de Janeiro, observaram alta concordância entre altura e peso aferidos e informados, este último, independentemente de idade, escolaridade, renda e estado nutricional. 
A amostra final do estudo de Pelotas constituiu-se de 508 adolescentes (254 casos e 254 controles), após contabilizar a perda de 10 pares por não identificação dos indivíduos casos ou controles para a etapa da entrevista. Em São Paulo, 42 adolescentes recusaram participação no estudo e outros quatro foram excluídos por diagnóstico de hipotireoidismo, sendo a amostra final constituída por 172 adolescentes (83 casos e 89 controles). Com perda de 73 adolescentes durante o estudo transversal, o estudo de Goiânia contou com amostra final de 223 adolescentes (106 casos e 117 controles). Informações mais detalhadas sobre as perdas amostrais dos estudos das três cidades podem ser encontradas nas respectivas publicações originais ${ }^{11-13}$.

Para fins da presente análise, os adolescentes definidos como controles (eutróficos) nos três estudos anteriores, permaneceram com a mesma classificação, com exceção dos $50 \%$ do estudo de Goiânia que foram eliminados por sorteio. Casos foram definidos como os indivíduos com sobrepeso, assim identificados nos três estudos citados, utilizando-se o critério de Must et al. ${ }^{15}$ em Pelotas e em Goiânia e a adaptação proposta pelos pesquisadores (IMC>percentil 89) em função dos objetivos do estudo de São Paulo.

Procedeu-se à rigorosa leitura e análise dos três questionários utilizados nos estudos acima descritos, constatando-se que esses instrumentos, apesar de similares no conteúdo questionado, apresentavam diferenças com relação ao tipo de algumas variáveis (contínuas/categóricas), ao conteúdo das respostas (por exemplo, diferenças nas categorias de resposta, no caso das variáveis categóricas) ou ao contexto da pergunta. Dessa forma, foram selecionadas para o estudo apenas as variáveis que apresentavam comparabilidade nesses quesitos, de modo a possibilitar a análise conjunta das mesmas.

Inicialmente procedeu-se à análise bivariada para observar as prevalências de exposição para casos e controles e testar as associações segundo o teste de Pearson $\left(\chi^{2}\right)$ e as razões de chance (Odds ratio) com seus respectivos intervalos de confiança (IC) com confiabilidade de $95 \%$. Em seguida foi utilizado o modelo de regressão logística para estimar riscos ajustados para o sobrepeso nos indivíduos da amostra ${ }^{19}$, segundo modelo conceitual com quatro níveis hierárquicos: 1) Condição socioeconômica (escolaridade materna); 2) História dos pais (IMC materno e paterno); 3) História do adolescente (peso ao nascer); 4) Estilos de vida do adolescente (tempo assistindo televisão, horas de sono noturno, consumo de frituras, de gordura visível da carne, de pele do frango e de fast food, consumo de álcool e hábito de fumar). De acordo com essa abordagem, a escolha dos fatores a serem incluídos no modelo multivariado não se baseia unicamente em associações estatísticas, mas em modelo conceitual que descreve as relações hierárquicas entre os fatores de risco que estão sendo estudados ${ }^{20}$. É importante salientar que apenas as variáveis comparáveis entre os três estudos compuseram os níveis hierárquicos, motivo pelo qual dois desses níveis estão representados por apenas uma variável.

Optou-se por testar todas as variáveis incluídas na análise bivariada na modelagem inicial, separadas por sexo. Os dois modelos finais (masculino e feminino) apresentam variáveis de todos os níveis hierárquicos propostos no modelo conceitual, dessa forma algumas variáveis permaneceram no modelo final, mesmo quando não associadas ao desfecho. A comparação de meninos e meninas foi feita considerando-se que os modelos apresentados foram ajustados independentemente, um para cada sexo.

\section{RESULTADOS}

Fizeram parte da análise 903 adolescentes (443 casos e 460 controles) com média de idade de 16,7 (1,3 Desvio-padrão - DP). Na Tabela 1 observa-se que a média de IMC das três cidades é bastante similar entre os controles, diferindo ligeiramente entre os casos. Para ambos os sexos, São Paulo apresenta a maior média, seguido de Goiânia e de Pelotas. A faixa de idade dos adoles- 
centes variou de 15 a 20 anos em Pelotas, 14 a 19 em São Paulo, e 16 a 20 em Goiânia.

Na Tabela 2 encontram-se a distribuição percentual (\%) dos fatores de risco para casos e controles, Odds ratio não-ajustado e respectivos intervalos de confiança, e teste de significância ( $\chi^{2}$ Pearson), segundo cada um dos estudos separadamente e para o três em análise combinada. Escolaridade materna menor do que 8 anos não se associou significantemente ao sobrepeso entre adolescentes, tanto na análise combinada como para as três cidades individualmente, apesar de ser possível observar uma tendência de risco entre adolescentes de São Paulo (associação limítrofe). Ressalta-se, no entanto, que a direção da associação, apesar de não significante, é contrária em Pelotas, quando comparada às outras duas cidades e à análise dos três bancos combinados. Sobrepeso $\left(25 \mathrm{~kg} / \mathrm{m}^{2}\right)$ e obesidade $\left(30 \mathrm{~kg} / \mathrm{m}^{2}\right)$ da mãe apresentaram associação significante em relação ao sobrepeso dos filhos nas três cidades e na análise combinada. O IMC paterno, por sua vez, apresentou comportamento bastante diverso: em Goiânia tanto sobrepeso como obesidade paternos não se associaram de forma significante ao excesso de peso nos adolescentes; em São Paulo somente obesidade paterna não esteve associada ao desfecho, enquanto que em Pelotas e na análise conjunta das três cidades o IMC dos pais aparece associado positivamente ao sobrepeso dos filhos. Ao analisar a influência do peso ao nascer dos adolescentes sobre o sobrepeso dos mesmos, apenas no grupo de Pelotas foi observada associação significante, o mesmo se repetindo para a variável tempo assistindo televisão (TV). Dormir nove ou menos horas durante a noite não se mostrou como fator risco para sobrepeso nos três estudos, considerados separada ou conjuntamente. Quanto ao comportamento alimentar, aos hábitos de consumir bebidas alcoólicas e de fumar, não foram observadas associações entre casos e controles nos três estudos analisados separadamente, mantendo-se essa resposta na análise combinada. Ainda que não significantes, destacam-se algumas tendências a direções contrárias no caso do consumo de pele de frango em Pelotas; do consumo de fast food e do fumo na amostra de São Paulo.

A Tabela 3 ilustra os resultados da análise bivariada por sexo, a partir da qual foram identificadas as variáveis que entraram no modelo final de regressão. Com a estratificação por sexo ficou evidenciada a associação positiva do IMC paterno e materno com o IMC dos adolescentes de ambos os sexos. Assistir televisão por mais de quatro horas associou-se positivamente ao sobrepeso em meninas, enquanto que as variáveis escolaridade materna e sono noturno apresentaram associação inversa ao excesso de peso entre as adolescentes do sexo feminino, tendência já observada na Tabela 2.

Na Tabela 4 apresentam-se os resultados das duas análises multivariadas por sexo, em que se utilizou uma variável de identificação dos três bancos de dados, para a qual o modelo foi ajustado a fim de ponderar os tamanhos amostrais nos efeitos encontrados.

Tabela 1. Distribuição do índice de massa corporal de adolescentes (casos e controles) de três cidades brasileiras (Pelotas [2000], São Paulo [2002] e Goiânia [2004]), segundo cidade de residência e sexo.

\begin{tabular}{|c|c|c|c|c|c|c|c|c|}
\hline \multirow{3}{*}{ Cidades de origem } & \multicolumn{4}{|c|}{ IMC $\left(\mathrm{kg} / \mathrm{m}^{2}\right)$} & \multicolumn{4}{|c|}{ IMC (kg/m²) } \\
\hline & Casos & \multirow{2}{*}{$\frac{\text { masculinos }}{\text { DP }}$} & \multicolumn{2}{|c|}{ Controles masculinos } & \multicolumn{2}{|c|}{ Casos femininos } & \multicolumn{2}{|c|}{ Controles femininos } \\
\hline & Média & & Média & DP & Média & DP & Média & DP \\
\hline Pelotas & 27,69 & 3,74 & 20,69 & 1,81 & 27,41 & 3,19 & 20,55 & 1,67 \\
\hline São Paulo & 28,95 & 2,65 & 20,17 & 2,01 & 30,40 & 2,85 & 20,26 & 1,91 \\
\hline Goiânia & 28,58 & 3,18 & 21,31 & 2,22 & 28,69 & 2,72 & 19,94 & 2,22 \\
\hline Total & 28,11 & 3,48 & 20,73 & 1,96 & 28,39 & 3,25 & 20,30 & 1,91 \\
\hline
\end{tabular}

DP: desvio-padrão. 
Tabela 2. Odds ratio e respectivos intervalos de confiança de características relacionadas ao sobrepeso (IMC $\geq$ percentil 85) entre adolescentes brasileiros das cidades de Pelotas [2000], São Paulo [2002] e Goiânia [2004].

\begin{tabular}{|c|c|c|c|c|c|c|c|c|c|c|}
\hline \multirow{3}{*}{ Variáveis } & \multicolumn{5}{|c|}{ Pelotas } & \multicolumn{5}{|c|}{ Sâo Paulo } \\
\hline & \multicolumn{2}{|c|}{$\begin{array}{l}\text { Casos } \\
\text { (sim) }\end{array}$} & \multicolumn{2}{|c|}{$\begin{array}{l}\text { Controles } \\
\text { (sim) }\end{array}$} & \multirow{2}{*}{$\begin{array}{c}\text { OR } \\
(\text { IC } 95 \%) \\
\end{array}$} & \multicolumn{2}{|c|}{$\begin{array}{c}\text { Casos } \\
\text { (sim) }\end{array}$} & \multicolumn{2}{|c|}{$\begin{array}{c}\text { Controles } \\
\text { (sim) }\end{array}$} & \multirow{2}{*}{$\begin{array}{c}\text { OR } \\
\text { (IC 95\%) } \\
\end{array}$} \\
\hline & $n$ & $\%$ & $\mathrm{n}$ & $\%$ & & $\mathrm{n}$ & $\%$ & $\mathrm{n}$ & $\%$ & \\
\hline \multicolumn{11}{|l|}{ Condição socioeconômica } \\
\hline Escolaridade materna $\leq 8$ anos & 18 & 7,1 & 22 & 8,7 & $0,8(0,4-1,6)$ & 26 & 31,7 & 18 & 20,2 & $1,8(0,9-3,9)$ \\
\hline \multicolumn{11}{|l|}{ História dos pais } \\
\hline IMC materno $\geq 25 \mathrm{~kg} / \mathrm{m}^{2}$ & 122 & 51,1 & 94 & 38,1 & $1,7(1,2-2,5)$ & 65 & 80,3 & 45 & 51,7 & $3,8(1,8-8,1)$ \\
\hline IMC paterno $\geq 25 \mathrm{~kg} / \mathrm{m}^{2}$ & 179 & 78,6 & 146 & 63,5 & $2,1(1,4-3,2)$ & 52 & 77,6 & 47 & 61,8 & $2,1(1,0-4,9)$ \\
\hline $\mathrm{IMC}$ materno $\geq 30 \mathrm{~kg} / \mathrm{m}^{2}$ & 41 & 17,2 & 19 & 7,7 & $2,5(1,4-4,7)$ & 31 & 38,3 & 19 & 21,8 & $2,2(1,1-4,6)$ \\
\hline IMC paterno $\geq 30 \mathrm{~kg} / \mathrm{m}^{2}$ & 64 & 28,1 & 31 & 13,5 & $2,5(1,5-4,2)$ & 20 & 29,9 & 13 & 17,1 & $2,1(0,9-5,0)$ \\
\hline \multicolumn{11}{|l|}{ História do adolescente } \\
\hline Peso ao nascer $\geq 4000 \mathrm{~g}$ & 47 & 18,5 & 31 & 12,2 & $1,6(1,0-2,8)$ & 6 & 6,7 & 10 & 12,1 & $1,9(0,6-6,6)$ \\
\hline \multicolumn{11}{|l|}{ Inatividade física } \\
\hline Tempo de TV $\geq 4$ horas/dia & 118 & 46,5 & 84 & 33,1 & $1,8(1,2-2,6)$ & 16 & 19,3 & 16 & 18,0 & $1,1(0,5-2,5)$ \\
\hline Sono noturno $\leq 9$ horas/dia & 197 & 77,6 & 184 & 72,5 & $1,3(0,9-2,0)$ & 59 & 71,1 & 61 & 68,5 & $1,1(0,6-2,3)$ \\
\hline \multicolumn{11}{|l|}{ Comportamento alimentar } \\
\hline Consumo de fritura $\geq 3$ vezes/semana & 48 & 18,9 & 51 & 20,1 & $0,9(0,6-1,5)$ & 31 & 37,4 & 36 & 40,9 & $0,9(0,4-1,7)$ \\
\hline Consumo de gordura visível da carne & 56 & 22,1 & 46 & 18,1 & $1,3(0,8-2,0)$ & 16 & 19,3 & 14 & 15,9 & $1,3(0,5-3,0)$ \\
\hline Consumo de pele do frango & 79 & 31,5 & 70 & 28,0 & $1,2(0,8-1,8)$ & 19 & 23,2 & 34 & 38,6 & $0,5(0,2-1,0)$ \\
\hline Consumo de fast food $\geq 3$ vezes/semana & 9 & 3,4 & 9 & 3,5 & $1,0(0,3-2,9)$ & 11 & 13,4 & 8 & 9,1 & $1,5(0,5-4,7)$ \\
\hline \multicolumn{11}{|l|}{ Hábitos de saúde } \\
\hline Consumo de álcool & 133 & 52,4 & 148 & 58,3 & $0,8(0,5-1,1)$ & 33 & 39,8 & 44 & 49,4 & $0,7(0,4-1,3)$ \\
\hline \multirow[t]{2}{*}{ Fumo } & 15 & 5,9 & 12 & 4,7 & $1,3(0,5-3,0)$ & 6 & 7,3 & 7 & 8,0 & $0,9(0,2-3,3)$ \\
\hline & \multicolumn{5}{|c|}{ Goiânia } & \multicolumn{5}{|c|}{ Pelotas + São Paulo + Goiânia } \\
\hline \multicolumn{11}{|l|}{ Condição socioeconômica } \\
\hline Escolaridade materna $\leq 8$ anos & 19 & 17,9 & 15 & 12,8 & $1,5(0,7-3,3)$ & 63 & 14,3 & 55 & 12,0 & $1,2(0,8-1,8)$ \\
\hline \multicolumn{11}{|l|}{ História dos pais } \\
\hline IMC materno $\geq 25 \mathrm{~kg} / \mathrm{m}^{2}$ & 65 & 62,5 & 43 & 37,7 & $2,8(1,5-4,9)$ & 252 & 59,4 & 182 & 40,6 & $2,1(1,6-2,8)$ \\
\hline IMC paterno $\geq 25 \mathrm{~kg} / \mathrm{m}^{2}$ & 67 & 68,4 & 68 & 61,8 & $1,3(0,7-2,5)$ & 298 & 75,8 & 261 & 62,7 & $1,9(1,4-2,6)$ \\
\hline IMC materno $\geq 30 \mathrm{~kg} / \mathrm{m}^{2}$ & 27 & 26,0 & 9 & 7,9 & $4,1(1,7-10,4)$ & 137 & 32,3 & 81 & 18,1 & $2,1(1,6-3,0)$ \\
\hline IMC paterno $\geq 30 \mathrm{~kg} / \mathrm{m}^{2}$ & 29 & 29,6 & 22 & 20,0 & $1,7(0,8-3,4)$ & 151 & 38,4 & 112 & 26,9 & $1,7(1,2-2,3)$ \\
\hline \multicolumn{11}{|l|}{ História do adolescente } \\
\hline Peso ao nascer $\geq 4000 \mathrm{~g}$ & 18 & 17,0 & 21 & 18,0 & $0,9(0,4-2,0)$ & 75 & 16,9 & 58 & 12,6 & $1,4(0,9-2,1)$ \\
\hline \multicolumn{11}{|l|}{ Inatividade física } \\
\hline Tempo de TV $\geq 4$ horas/dia & 35 & 33,0 & 31 & 26,5 & $1,4(0,7-2,5)$ & 169 & 38,2 & 131 & 28,5 & $1,5(1,2-2,1)$ \\
\hline Sono noturno $\leq 9$ horas/dia & 70 & 66,0 & 79 & 67,5 & $0,9(0,5-1,7)$ & 326 & 73,6 & 324 & 70,4 & $1,2-(0,9-1,6)$ \\
\hline Comportamento alimentar & & & & & & & & & & \\
\hline Consumo de fritura $\geq 3$ vezes/semana & 41 & 38,7 & 54 & 46,2 & $0,7(0,4-1,3)$ & 120 & 27,1 & 141 & 30,7 & $0,8(0,6-1,1)$ \\
\hline Consumo de gordura visível da carne & 33 & 31,1 & 36 & 30,8 & $1,0(0,6-1,9)$ & 105 & 23,7 & 96 & 20,9 & $1,2(0,8-1,6)$ \\
\hline Consumo de pele do frango & 34 & 32,1 & 40 & 34,2 & $0,9(0,5-1,7)$ & 132 & 30,1 & 144 & 31,7 & $0,9(0,7-1,2)$ \\
\hline Consumo de fast food $\geq 3$ vezes/semana & 24 & 22,6 & 33 & 28,2 & $0,7(0,4-1,4)$ & 44 & 10,0 & 50 & 10,9 & $0,9(0,6-1,4)$ \\
\hline Hábitos de saúde & & & & & & & & & & \\
\hline Consumo de álcool & 49 & 46,2 & 56 & 47,9 & $0,9(0,5-1,6)$ & 215 & 48,5 & 248 & 53,9 & $0,8(0,6-1,1)$ \\
\hline Fumo & 10 & 9,4 & 8 & 6,8 & $1,4(0,5-4,3)$ & 31 & 7,0 & 27 & 5,9 & $1,2(0,7-2,1)$ \\
\hline
\end{tabular}

*de cada uma das cidades e das três cidades combinadas

OR: odds ratio; IC: intervalo de confiança; IMC: índice de massa corporal; OR significantes destacados em negrito. 
510 | I.C. RIBEIRO et al.

Tabela 3. Características relacionadas ao sobrepeso (IMC $\geq$ percentil 85) entre adolescentes do sexo masculino e feminino moradores de Pelotas [2000], São Paulo [2002] e Goiânia [2004], odds ratio não ajustado e respectivos intervalos de confiança referentes aos bancos das três cidades combinados.

\begin{tabular}{|c|c|c|c|c|c|c|c|c|c|c|c|c|}
\hline \multirow{3}{*}{$\begin{array}{l}\text { Variáveis de acordo com } \\
\text { modelo hierárquico }\end{array}$} & \multicolumn{6}{|c|}{ Masculino } & \multicolumn{6}{|c|}{ Feminino } \\
\hline & \multicolumn{2}{|c|}{ Casos } & \multicolumn{2}{|c|}{ Controles } & \multirow{2}{*}{ OR (IC 95\%) } & \multirow{2}{*}{$p$ valor ${ }^{*}$} & \multicolumn{2}{|c|}{ Casos } & \multicolumn{2}{|c|}{ Controles } & \multirow{2}{*}{ OR (IC 95\%) } & \multirow{2}{*}{$p$ valor* } \\
\hline & $n$ & $\%$ & $\mathrm{n}$ & $\%$ & & & $\mathrm{n}$ & $\%$ & $n$ & $\%$ & & \\
\hline \multicolumn{13}{|l|}{ Condição socioeconômica } \\
\hline $\begin{array}{l}\text { Escolaridade materna } \leq 8 \\
\text { anos }\end{array}$ & 25 & 9,8 & 27 & 11,0 & $0,9(0,5-1,6)$ & 0,667 & 38 & 20,2 & 28 & 13,0 & $1,7(1,0-2,9)$ & 0,052 \\
\hline \multicolumn{13}{|l|}{ História dos pais } \\
\hline IMC materno $\geq 25 \mathrm{Kg} / \mathrm{m}^{2}$ & 134 & 54,7 & 99 & 41,6 & $1,7(1,2-2,5)$ & 0,004 & 118 & 65,9 & 83 & 39,5 & $3,0(1,9-4,6)$ & 0,000 \\
\hline $\mathrm{IMC}$ paterno $\geq 25 \mathrm{Kg} / \mathrm{m}^{2}$ & 181 & 78,7 & 142 & 64,0 & $2,1(1,3-3,2)$ & 0,001 & 117 & 71,8 & 119 & 61,3 & $1,6(1,0-2,6)$ & 0,038 \\
\hline IMC materno $\geq 30 \mathrm{Kg} / \mathrm{m}^{2}$ & 49 & 20,0 & 26 & 10,9 & $2,0(1,2-3,6)$ & 0,002 & 21 & 10,0 & 6 & 9,5 & $3,5(1,9-6,4)$ & 0,000 \\
\hline IMC paterno $\geq 30 \mathrm{Kg} / \mathrm{m}^{2}$ & 65 & 28,2 & 39 & 17,6 & $1,8(1,2-3,0)$ & 0,007 & 48 & 29,5 & 27 & 13,9 & $2,6(1,5-4,6)$ & 0,000 \\
\hline \multicolumn{13}{|l|}{ História do adolescente } \\
\hline $\begin{array}{l}\text { Peso ao nascer } \geq 4000 \mathrm{~g} \\
\text { Inatividade física }\end{array}$ & 51 & 20,1 & 40 & 16,3 & $1,3(0,8-2,1)$ & 0,278 & 24 & 12,7 & 22 & 10,2 & $1,3(0,7-2,5)$ & 0,436 \\
\hline Tempo de TV $\geq 4$ horas/dia & 103 & 40,5 & 82 & 33,5 & $1,4(0,9-2,0)$ & 0,102 & 66 & 34,9 & 49 & 20,8 & $1,8(1,1-2,9)$ & 0,007 \\
\hline Sono noturno $\leq 9$ horas/dia & 190 & 74,8 & 190 & 75,5 & $0,9(0,6-1,3)$ & 0,471 & 130 & 72,0 & 134 & 62,3 & $1,6(1,0-2,4)$ & 0,040 \\
\hline \multicolumn{13}{|l|}{ Comportamento alimentar } \\
\hline $\begin{array}{l}\text { Consumo de frituras } \geq 3 \\
\text { vezes por semana }\end{array}$ & 79 & 31,1 & 81 & 33,1 & $0,9(0,6-1,4)$ & 0,639 & 41 & 21,7 & 60 & 28,0 & $0,7(0,4-1,1)$ & 0,142 \\
\hline $\begin{array}{l}\text { Consumo de gordura } \\
\text { visível da carne = Sim }\end{array}$ & 78 & 30,7 & 59 & 24,1 & $1,4(0,9-2,1)$ & 0,097 & 27 & 14,3 & 37 & 17,3 & $0,8(0,4-1,4)$ & 0,410 \\
\hline $\begin{array}{l}\text { Consumo de pele do } \\
\text { frango }=\operatorname{Sim}\end{array}$ & 100 & 39,7 & 90 & 37,3 & $1,1(0,8-1,6)$ & 0,594 & 32 & 17,1 & 54 & 25,2 & $0,6(0,4-1,0)$ & 0,048 \\
\hline $\begin{array}{l}\text { Consumo de fast food } \geq 3 \\
\text { vezes por semana }\end{array}$ & 29 & 11,5 & 33 & 13,5 & $0,8(0,5-1,5)$ & 0,498 & 15 & 7,9 & 17 & 7,9 & $1,0(0,4-2,2)$ & 0,998 \\
\hline \multicolumn{13}{|l|}{ Hábitos de saúde } \\
\hline Consumo de álcool = Sim & 126 & 49,6 & 146 & 59,6 & $0,7(0,5-0,9)$ & 0,025 & 89 & 47,1 & 102 & 47,4 & $1,0(0,7-1,5)$ & 0,944 \\
\hline Fumo $=$ Sim & 17 & 6,7 & 13 & 5,3 & $1,3(0,6-2,9)$ & 0,515 & 14 & 7,5 & 14 & 6,5 & $1,1(0,5-2,7)$ & 0,722 \\
\hline
\end{tabular}

IMC: índice de massa corporal; OR: odds ratio; IC: intervalo de confiança.

${ }^{*} p$ valor correspondente ao teste do $\chi^{2}$ de Pearson. OR significantes destacados em negrito.

A primeira variável a entrar em ambos os modelos foi escolaridade materna, que se manteve não associada ao sobrepeso dos adolescentes do sexo masculino, como já observado na análise bivariada. Por outro lado, o risco de adolescentes do sexo feminino apresentarem sobrepeso, quando filhas de mães com menos de 8 anos de escolaridade, é duas vezes maior do que entre aquelas cujas mães estudaram mais de 8 anos. Optou-se por deixar no modelo apenas as variáveis obesidade materna e paterna, dada a maior magnitude da associação destas duas variáveis, quando comparadas ao sobrepeso materno e paterno. Tanto obesidade da mãe como do pai, mesmo após o ajuste, permanecem associadas ao sobrepeso dos filhos. Entre os meninos, ter pai com IMC igual ou acima de $30 \mathrm{~kg} / \mathrm{m}^{2}$ duplica o risco de sobrepeso, enquanto ter mãe obesa representa 2,6 mais chances de ter sobrepeso quando comparados aos filhos de pais com IMC abaixo de $30 \mathrm{~kg} / \mathrm{m}^{2}$. Para as meninas essa associação é ainda mais forte, sendo as chances 3,3 vezes e 2,3 vezes maiores de ter sobrepeso quando suas mães ou pais são obesos, em comparação àquelas com mães ou pais não obesos.

O peso ao nascer, quando controlado pelas variáveis anteriores, continua não associado ao sobrepeso entre os indivíduos de ambos os sexos 
Tabela 4. Modelos hierárquicos de regressão logística de fatores de risco para sobrepeso em adolescentes de três cidades brasileiras (Pelotas [2000] + São Paulo [2002] + Goiânia [2004]) do sexo masculino e feminino.

\begin{tabular}{|c|c|c|c|c|c|c|}
\hline \multirow{2}{*}{ Variável } & \multicolumn{3}{|c|}{ Masculino } & \multicolumn{3}{|c|}{ Feminino } \\
\hline & OR ajustado (95\%IC) & $p$-valor ${ }^{*}$ & Modelos $^{* *}$ & OR ajustado (95\%IC) & $p$-valor ${ }^{*}$ & Modelos $^{* * *}$ \\
\hline \multicolumn{7}{|l|}{ Condição socioeconômica } \\
\hline Escolaridade materna $\leq 8$ anos & $0,8(0,4-1,6)$ & 0,57 & 1 & $2,3(1,2-4,4)$ & 0,02 & 1 \\
\hline \multicolumn{7}{|l|}{ História dos pais } \\
\hline $\mathrm{IMC}$ materno $\geq 30 \mathrm{Kg} / \mathrm{m}^{2}$ & $2,6(1,5-4,6)$ & 0,00 & 2 & $3,3(1,8-6,3)$ & 0,00 & 2 \\
\hline $\mathrm{IMC}$ paterno $\geq 30 \mathrm{Kg} / \mathrm{m}^{2}$ & $1,9(1,2-3,1)$ & 0,00 & 2 & $2,3(1,3-4,1)$ & 0,00 & 2 \\
\hline \multicolumn{7}{|l|}{ História do adolescente } \\
\hline Peso ao nascer $\geq 4000 \mathrm{~g}$ & $1,2(0,7-2,1)$ & 0,42 & 3 & $1,3(0,6-2,7)$ & 0,45 & 3 \\
\hline \multicolumn{7}{|l|}{ Inatividade física } \\
\hline Tempo de TV $\geq 4$ horas/dia & $1,3(0,9-2,0)$ & 0,21 & & $1,7(1,0-2,8)$ & 0,04 & 4 \\
\hline Sono noturno $\leq 9$ horas & $0,9(0,6-1,4)$ & 0,61 & & $1,6(1,0-2,5)$ & 0,07 & 4 \\
\hline \multicolumn{7}{|l|}{ Comportamento alimentar } \\
\hline $\begin{array}{l}\text { Consumo de gordura } \\
\text { visível da carne }\end{array}$ & $1,7(1,1-2,6)$ & 0,03 & 4 & $0,6(0,3-1,2)$ & 0,16 & \\
\hline $\begin{array}{l}\text { Consumo de frituras } \geq 3 \\
\text { vezes/semana }\end{array}$ & $0,7(0,5-1,1)$ & 0,11 & & $0,5(0,3-0,9)$ & 0,03 & 4 \\
\hline \multicolumn{7}{|l|}{ Hábitos de saúde } \\
\hline Consumo de álcool & $0,6(0,4-0,9)$ & 0,02 & 4 & $0,9(0,5-1,4)$ & 0,54 & \\
\hline
\end{tabular}

OR: odds ratio; IC: intervalo de confiança; IMC: índice de massa corporal.

OR e $p$-valor significantes destacados em negrito.

* $p$-valor do Teste da Razão de Verossimilhança.

** Masculino Modelo 1 - Escolaridade materna; Modelo 2 - obesidade (IMC $\geq 30 \mathrm{Kg} / \mathrm{m}^{2}$ ) materna e paterna ajustadas entre si + Modelo 1; Modelo

3 - Modelo 2 adicionado do peso ao nascer; Modelo 4 - Modelo 3 + consumo de gordura visível da carne, consumo de álcool.

${ }^{* * *}$ Feminino Modelo 1 - Escolaridade materna; Modelo 2 - obesidade (IMC $\geq 30 \mathrm{Kg} / \mathrm{m}^{2}$ ) materna e paterna ajustadas entre si + Modelo 1; Modelo

3 - Modelo 2 adicionado do peso ao nascer; Modelo 4 - Modelo 3 + tempo de TV, sono noturno, consumo de frituras.

estudados. Após ajuste pelos três níveis anteriores, o hábito de assistir TV por mais de 4horas/dia e a duração do sono noturno menor ou igual a 9 horas representam riscos, respectivamente $70 \%$ e $60 \%$ maiores de adolescentes do sexo feminino apresentarem sobrepeso, em comparação com as colegas que permanecem menos de 4 horas por dia assistindo TV ou que dormem mais de 9 horas por noite. Entre os meninos, nenhuma das duas variáveis esteve significantemente associada ao excesso de peso dos adolescentes. Quanto aos hábitos alimentares, a análise ajustada revelou associações significantes; positiva $(O R=1,7)$ entre o consumo de gordura aparente de carne vermeIha e o excesso de peso em meninos, e negativa $(\mathrm{OR}=0,5)$ entre o consumo freqüente de frituras e o sobrepeso de meninas. Finalmente, o consumo de álcool aparece inversamente associado $(\mathrm{OR}=0,6)$ ao sobrepeso de adolescentes do sexo masculino.

\section{S C U S S Ã O}

Ao possibilitar a utilização de uma amostra maior, a análise combinada (conjunta) de bancos de dados, oferece a oportunidade de examinar variações nas associações entre subgrupos populacionais, proporcionando maior poder estatístico do que nos estudos individualizados ${ }^{14}$. A apresentação dos dados por sexo visa atender às possíveis diferenças decorrentes do desenvolvimento puberal e do nível de atividade física que possam refletir em mudanças na composição corporal no período da adolescência. A análise multivariada hierár- 
quica possibilitou a estimativa dos riscos que representam de cada variável, controlando concomitantemente possíveis fatores de confusão. Nenhuma das variáveis cujos valores de Odds brutos foram significantes perdeu efeito após o ajuste no modelo. Ao contrário, com a abordagem utilizada foi possível identificar novos efeitos sobre o desfecho estudado.

É possível que um indicador direto como a renda familiar fosse mais adequado para avaliar a relação entre o estado nutricional de adolescentes e o nível socioeconômico da família. Entretanto, vários autores ${ }^{21,22}$ têm utilizado a escolaridade de ambos ou de um dos pais como indicador indireto da situação socioeconômica familiar. Escolaridade dos pais mede também possibilidade de assimilação e utilização de novos conhecimentos pela família. No presente estudo observou-se forte associação entre o sobrepeso em meninas e a menor escolaridade ( $\leq 8$ anos) das suas mães. Moreno et al. ${ }^{22}$, em análise de amostra nacional de crianças e adolescentes espanhóis, observaram associação inversa entre escolaridade materna e sobrepeso em adolescentes do sexo masculino, mas não entre meninas. As chances de apresentarem sobrepeso aumentam em $70 \%$ quando os adolescentes são filhos de mães que só freqüentaram a escola elementar. Um estudo prospectivo (National Longitudinal Survey of Youth), com amostra nacional de crianças americanas acompanhadas durante seis anos, revelou que os filhos de mães com ensino fundamental incompleto apresentavam risco 50\% maior de desenvolverem sobrepeso do que aqueles filhos de mães com maior nível de escolaridade. No entanto, na análise ajustada, esse risco mostrou-se dependente de outras variáveis tais como IMC materno e indicadores sócio-demográficos ${ }^{23}$.

Em concordância com outros estudos epidemiológicos ${ }^{21,24}$, observou-se que ser filho de pais com $I M C \geq 30 \mathrm{~kg} / \mathrm{m}^{2}$ é importante fator de risco para sobrepeso entre adolescentes. A associação mais forte entre o sobrepeso entre adolescentes de ambos os sexos e o IMC materno, em relação ao IMC paterno, sugere que o estado nutricional dos adolescentes, apesar de ter um importante componente genético, é fortemente determinado pelo ambiente e pelas práticas alimentares da família, essencialmente hábitos maternos, processo facilitado pelo maior envolvimento das mães com o crescimento e o desenvolvimento dos filhos, em especial na escolha e no preparo dos alimentos. Estudos longitudinais apontam para uma associação positiva entre peso ao nascer e adiposidade na infância e na vida adulta ${ }^{25,26}$. Na presente análise não foi observada associação entre peso ao nascer superior a $4000 \mathrm{~g}$ e sobrepeso na adolescência nos modelos finais de ambos os sexos. Essa associação pode ser mediada pelo estado nutricional dos pais, ou seja; bebês grandes não serão, necessariamente, crianças e adolescentes obesos, a menos que a mãe ou o pai (ou ambos) sejam também obesos e/ou proporcionem um ambiente favorável à obesidade²7.

Não está ainda claramente definida a seqüência causal entre assistir muitas horas de televisão e sobrepeso entre adolescentes. Seria o padrão sedentário de vida que causaria a obesidade ou o estigma social sofrido por obesos que determinaria o isolamento e sedentarismo? Vários autores vêm, no entanto, referindo associação positiva entre tempo assistindo televisão e sobrepeso em crianças e adolescentes ${ }^{28-30}$. No presente estudo a análise não-ajustada revelou associação significante entre sobrepeso e tempo assistindo $T V \geq 4 h / d i a$ no grupo de adolescentes de Pelotas e em relação aos três bancos combinados, mas não entre moradores de São Paulo e Goiânia, áreas em que, por apresentarem maior densidade populacional11' esperava-se confirmar a associação entre sedentarismo, urbanização e sobrepeso/obesidade em crianças e adolescentes ${ }^{29}$. Ao separar os adolescentes por sexo no modelo final fica evidente a participação dessa variável (tempo TV) na determinação do sobrepeso entre as meninas. Nessa mesma linha, Hallal et al..$^{30} \mathrm{em}$ estudo com adolescentes de 10 a 12 anos participantes da Coorte de Nascimentos de Pelotas, encontraram maior percentual de atividade física de lazer entre meninos do que entre meninas, e uma relação inversa entre o tempo assistindo TV 
e o percentual de atividade física de lazer entre os meninos.

Pode-se afirmar que o tempo de sono noturno entre adolescentes é parcialmente determinado por fatores sociais tais como acordar cedo para ir à escola e atividades que podem prosseguir até tarde da noite, como assistir televisão, jogar video-game e usar internet ${ }^{30}$. No presente estudo observou-se risco aumentado para sobrepeso entre meninas que relataram dormir menos de 9 horas por noite, condição não observada para os meninos. Knutson ${ }^{31}$, analisando dados de amostra nacional do Estudo Longitudinal sobre Saúde de Adolescentes Americanos (National Longitudinal Survey of Youth), relata resultados similares em associação inversa para meninos e não para meninas. Para meninos, cada hora a mais de duração do sono noturno corresponde à redução de 10\% no risco de sobrepeso.

A relação entre consumo alimentar e obesidade, dificilmente, é evidenciada em estudos transversais ${ }^{2}$. No presente estudo o consumo de frituras referido foi mais freqüente entre adolescentes do sexo feminino com peso normal do que entre aquelas com sobrepeso. Comparando o consumo alimentar em amostra probabilística de adolescentes com e sem sobrepeso, moradores da cidade do Rio de Janeiro, Andrade et al. ${ }^{2}$ observaram também maior consumo energético de gordura total entre meninos eutróficos do que entre aqueles com sobrepeso. Entre as meninas, o consumo de gorduras foi ligeiramente mais elevado no grupo com sobrepeso do que no grupo de peso normal, no entanto, é provável que esses achados reflitam um relato sob influência de informação prévia sobre as características de uma alimentação saudável ${ }^{2}$. Jebb ${ }^{32}$ refere que indivíduos com sobrepeso em geral tendem a relatar menor ingestão de alimentos do que a realidade, o que, em parte, responderia pelo número reduzido de estudos epidemiológicos demonstrando associação entre ingestão energética e peso corporal. Por outro lado, o consumo de alimentos ricos em gorduras e pouco saudáveis tornou-se a regra entre crianças e adolescentes, dificultando a identificação da já complexa relação entre estado nutricional e ingestão de alimentos.

Ainda que o resultado deste estudo demonstre o consumo de gordura visível da carne como fator de risco associado ao sobrepeso entre meninos, não foi possível caracterizar a magnitude da contribuição desse hábito na ingestão energética total e, conseqüentemente, no balanço energético diário dos adolescentes. O hábito de consumir carne vermelha é, em geral, associado ao sexo masculino na sociedade, o que pode ter contribuído para o relato de maior ingestão de gordura da carne entre os meninos do que entre as meninas no presente estudo (Tabela 3). Corroborando essa afirmação, os resultados de análise de inquérito alimentar com questionário auto-aplicado por estudantes de 11 a 18 anos de Santo André (SP), conduzida por Gambardella et al. ${ }^{33}$ revelaram maior consumo de carnes durante a refeição almoço entre os meninos do que entre as meninas. Finalmente, diante da associação inversa entre o consumo de álcool e o sobrepeso entre meninos, e da provável inexistência, de literatura que suporte esse achado, especula-se que essa relação reflita oportunidades de socialização menos freqüentes entre os adolescentes com sobrepeso, talvez decorrente de rejeição social sofrida pelo adolescente ${ }^{11}$.

Algumas limitações do presente estudo devem ser consideradas. Primeiramente, utilizou-se apenas um indicador de adiposidade (IMC $\geq \mathrm{p} 85$ ) entre os adolescentes, ao invés de associá-lo a outras medidas como circunferência da cintura e pregas cutâneas. No entanto, o IMC tem sido associado às medidas de adiposidade em crianças e em adolescentes de forma consistente entre as diferentes faixas etárias e para ambos os sexos ${ }^{34}$. Outra limitação foi a utilização de diferentes pontos de corte para a definição de sobrepeso entre os escolares de São Paulo, em relação ao utilizado em Pelotas e Goiânia e, por último, por ser este um estudo de natureza transversal, o mesmo permitiu determinar associações entre a variável dependente e as variáveis independentes, mas não estabelecer a seqüência temporal. 


\section{O N CLUS Ã O}

Conclui-se que o delineamento caso-controle contribuiu para elucidar alguns questionamentos a respeito dos determinantes do sobrepeso em adolescentes brasileiros, além de trazer novas questões para investigações futuras. A identificação de características que possam delimitar grupos de risco é essencial para a otimização de recursos humanos e financeiros em políticas e programas de combate ao sobrepeso e à obesidade entre adolescentes. Meninas provenientes de famílias com baixa escolaridade materna perecem ser um grupo chave na obtenção de acesso mais eficiente a informações e serviços de apoio e prevenção do ganho de peso, tais como promoção de hábitos alimentares saudáveis e prática de atividade física diária.

\section{A GRADECIMENTOS}

A Marilda Neutzling, Daniela Silveira e Ida Menezes, por fornecerem os bancos de dados e pela plena disponibilidade em atender os questionamentos dos autores. À Coordenação de Aperfeiçoamento de Pessoal de Nível Superior, pelo suporte financeiro ao primeiro autor durante estágio de doutorado no exterior (processo nº 3540/7); à Fundação e Amparo à Pesquisa do Estado de São Paulo, pelo apoio financeiro (processo no 03/00415-4) e ao Dr. Michael Pratt (PAHB/DNPA/CDC), pelo apoio e incentivo.

\section{COLABORADORES}

I.C. RIBEIRO participou da construção dos três bancos de dados, da análise, da discussão dos dados e da redação do artigo. F.A.B COLUGNATI participou da combinação dos bancos de dados em um banco único, da análise e da discussão dos resultados. J.A.A.C. TADDEl coordenou os projetos que deram origem aos três bancos de dados, participou da concepção do trabalho, da análise e da discussão dos resultados.

\section{REFERÊ NCIAS}

1. Spear BA. Adolescent growth and development. Am J Diet Assoc. 2002; (3 Suppl):S23-9.
2. Andrade RG, Pereira RA, Sichieri R. Consumo alimentar de adolescentes com e sem sobrepeso do Município do Rio de Janeiro. Cad Saúde Pública. 2003; 19(5):1485-95.

3. World Health Organization. Obesity: preventing and managing the global epidemic. Report of a WHO Consultation on Obesity. Geneva: WHO; 1998.

4. Taddei JA, Colugnati FB, Rodrigues EM, Sigulem DM, Lopez FA. Desvios nutricionais em menores de cinco anos. São Paulo: Unifesp; 2002.

5. Veiga GV, Cunha AS, Sichieri R. Trends in overweight among adolescents living in the poorest and richest regions of Brazil. Am J Public Health. 2004; 94(9):1544-8.

6. Instituto Brasileiro de Geografia e Estatística. Pesquisa de Orçamentos Familiares 2002-2003. Tendência secular do estado nutricional. Gráfico 28: indicadores antropométricos para adolescentes entre 10 e 19 anos de idade por sexo. Rio de Janeiro: Ministério da Saúde; 2006.

7. Banco Mundial. Enfrentando o desafio das doenças não transmissíveis no Brasil. Relatório $n$. 32576-BR. Brasília; 2005.

8. Finkelstein EA, Fiebelkorn IC, Wang G. National medical spending attributable to overweight and obesity: how much, and who's paying? Health Aff (Millwood). 2003; Suppl Web Exclusives:W3-26.

9. Sichieri R, Nascimento S. O Custo da obesidade para o Sistema Único de Saúde: perspectivas para as próximas décadas. In: Taddei JAAC, coordenador. Jornadas científicas do NISAN 2004/2005. São Paulo: Manole; 2006. p.101-11.

10. Instituto Brasileiro de Geografia e Estatística. Cidades@. [acesso 20 out 2006]. Disponível em: <http://www.ibge.gov.br/cidadesat/default.php>.

11. Neutzling MB, Taddei JAAC, Gigante DP. Risk factors for obesity among Brazilian adolescents: a case-control study. Public Health Nutr. 2003; 6(8): 743-9.

12. Silveira D, Taddei JA, Escrivão MA, Oliveira FL, Ancona-Lopez F. Risk factors for overweight among Brazilian adolescents of low-income families: a case-control study. Public Health Nutr. 2006; 9(4): 421-8.

13. Menezes IHCF. Fatores de risco para sobrepeso e obesidade em adolescentes ingressos na Universidade Federal de Goiás em 2004 [tese]. São Paulo: Universidade Federal de São Paulo; 2006.

14. Smith-Warner SA, Spiegelman D, Ritz J, Albanes $D$, Beeson WL, Bernstein L, et al. Methods for pooling results of epidemiologic studies: the Pooling Project of Prospective Studies of Diet and Cancer. Am J Epidemiol. 2006; 163(11):1053-64. 
15. Must A, Dallal GE, Dietz WH. Reference data for obesity: 85th and 95th percentiles of body mass index (wt/ht $\left.{ }^{2}\right)$ and triceps skinfold thickness. Am J Clin Nutr. 1991; 53(4):839-46. [Errata Am J Clin Nutr. 1991; 54(5):773].

16. World Health Organization. Physical status: the use and interpretation of anthropometry. Geneva: WHO; 1995. Report Series n. 854.

17. Nieto-García FJ, Bush TL, Keyl PM. Body mass definitions of obesity: sensitivity and specificity using self-reported weight and height. Epidemiology. 1990; 1(2):146-52.

18. Fonseca MJM, Faerstein E, Chor D, Lopes CS. Validade de peso e estatura informados e índice de massa corporal: estudo pró-saúde. Rev Saúde Pública. 2004; 38(3):392-8.

19. Hosmer DW, Lemeshow S. Applied logistic regression. New York: Wiley \& Sons; 1989.

20. Victora CG, Huttly SR, Fuchs SC, Olinto MTA. The role of conceptual frameworks in epidemiological analysis: a hierarchical approach. Int J Epidemiol. 1997; 26(1):224-7.

21. Marins VMR, Almeida RMVR, Pereira RA, Barros MBA. The relationship between parental nutritional status and overweight children/adolescents in Rio de Janeiro, Brazil. Public Health. 2004; 118(1): 43-9.

22. Moreno LA, Tomas C, Gonzalez-Gross M, Bueno G, Perez-Gonzalez JM, Bueno M. Micro-environmental and socio-demographic determinants of childhood obesity. Int J Obes Relat Metab Disord. 2004; 28(Suppl 3):S16-S20.

23. Strauss RS, Knight J. Influence of the home environment on the development of obesity in children. Pediatrics. 1999; 103(6):e85.

24. Maffeis C. Aetiology of overweight and obesity in children and adolescents. Eur J Pediatr. 2000; 159(Suppl 1):S35-S44.
25. Parsons TJ, Power C, Logan S, Summerbell CD. Childhood predictors of adult obesity: a systematic review. Int J Obes. 1999; 23(S8):S1-S41.

26. Oken E, Gillman MW. Fetal origins of obesity. Obes Res. 2003; 11(4):496-506.

27. Frisancho R. Prenatal compared with parental origins of adolescent fatness. Am J Clin Nutr. 2000; 72(5):1186-90.

28. Ribeiro IC, Taddei JAAC, Colugnati F. Obesity among children attending elementary public schools in São Paulo, Brazil: a case-control study. Public Health Nutr. 2003; 6(7):659-63.

29. Popkin BM. Urbanization, lifestyles changes and the nutrition transition. World Dev. 1999; 27(11): 1905-16.

30. Hallal PC, Bertoldi AD, Gonçalves H, Victora CG. Prevalência de sedentarismo e fatores associados em adolescentes de 10-12 anos de idade. Cad Saúde Pública. 2006; 22(6):1277-87.

31. Knutson KL. Sex differences in the association between sleep and body mass index in adolescents. J Pediatr. 2005; 147(6):830-4.

32. Jebb SA. Energy intake and body weight. In: Fairburn CG, Brownell KD, editors. Eating disorders and obesity: a comprehensive handbook. 2nd ed. New York: Guilford; 2002. p.37-42.

33. Gambardella AMD, Frutuoso MFP, Franch C. Prática alimentar de adolescentes. Rev Nutr. 1999; 12(1): 55-63.

34. Pietrobelli A, Faith MS, Allison DB, Gallagher D, Chiumello G, Heymsfield SB. Body mass index as a measure of adiposity among children and adolescents: a validation study. J Pediatr. 1998; 132(2):191-3.

Recebido em: 28/9/2007

Versão final reapresentada em: 17/3/2008 Aprovado em: 11/7/2008 